\title{
Reconstitution of the Torpedo californica nicotinic acetylcholine receptor into planar lipid bilayers
}

\author{
Theo Schürholz, Jürgen Weber and Eberhard Neumann \\ Department of Physical and Biophysical Chemistry, Faculty of Chemistry, University of Bielefeld, \\ P.O. Box 8640, D.4800 Bielefeld I (F.R. Germany)
}

(Received 26 May 1988; in revised form 1 November 1988)

\begin{abstract}
Detergent-solubilized acetylcholine receptor ( $\mathrm{nAcChR}$ ) proteins can be purified by affinity chromatography and reconstituted into lipid vesicles and afterwards into planar lipid bilayer membranes via, in principle, two methods: fusion or assembly from two vesicle-spread monolayers. In the presence of agonists (carbamoylcholine, suberoyldicholine) different kinds of channel openings are recorded: fast single channels, bursts and long openings with short closures in between. Similar results have been obtained with reconstituted membrane fragments rich in $\mathrm{nAcChR}$. In addition, Torpedo californica $\mathrm{nAcChR}$ proteins give rise to fuzzy channels and less defined events of conductivity, which "reemerge" again all the time. Frequently the channel events have conductance levels of about 200 to $300 \mathrm{pS}$, obviously simultaneous openings of several aggregated receptors. Under these conditions $40 \mathrm{pS}$ conductance events occur also. It appears that the conductance of the channels measured is a multiple of $6.3 \mathrm{pS}$. Often, with the same sample, no channel openings are seen. Contrary to patch-clamp investigations on whole cells, AcChR-channel openings in reconstituted systems occur only several minutes after agonist application and not immediately. It is not clear whether the "reconstituted channels" reflect rapid activation or whether they result from desensitized receptor states only. Although a clear-cut correlation of channel event and channel protein unit is only possible by reconstitution of the biochemically characterized protein, e.g. monomer, dimer or higher oligomers, the reconstitution technique is still in its infancy.
\end{abstract}

\section{INTRODUCTION}

The nicotinic actylcholine receptor (nAcChR) is the biochemically best characterized receptor-protein complex. However, functional reconstitution of $\mathrm{nAcChR}$ proteins into planar lipid bilayers has not been obtained satisfactorily, yet.

Nevertheless, the nAcChR has become the prototype of a chemically gated channel protein, being available in large quantities in the electric organ of Torpedo 
californica and other electric fishes. The $\mathrm{nAcChRs}$ from $T$. californica and $T$. marmorata have been purified as a light form or monomer of relative molecular mass $M_{\mathrm{r}}=290,000$ (of subunit composition $\alpha_{2} \beta \gamma \delta$, which are four homologous glycopeptides) and as a dimer of $M_{\mathrm{r}}=580,000$; the amino acid sequences of the AcChR subunits have been elucidated recently $[1,2]$. Information about the channel function of the muscle $\mathrm{nAcChR}$ has been provided by patch clamp recording [3-6].

The detailed biochemical and structural characterization of the $\mathrm{nAcChR}$ has served as a basis for the reconstitution of the purified receptors aimed at the investigation of the structure-function relationship, by combining molecular biochemical knowledge with functional-biophysical results. After reconstitution of AcChRs into planar bilayers the current through single channels was measured at a high time resolution [7-9]. Therefore it is possible, for example, to decide whether all the channels have the same size or if they show larger differences in conductance or gating behavior.

No channel activity was seen when nAcChRs were incorporated into lipid bilayers containing decane [10]. Boheim et al. [9] report the successful reconstitution of purified $\mathrm{AcChR}$ molecules by fusion of $\mathrm{AcChR}$ containing vesicles into virtually solvent-free lipid bilayers; hexadecane was used only in the pretreatment. Most of the data reported on reconstituted AcChRs were obtained by spreading protein-lipid vesicles to a monolayer and subsequent bilayer formation [11-13, and references therein]. Furthermore, vesicles with incorporated AcChRs were patched directly [14].

Although the authors of refs. 7-9 and 11-13 claim that the conditions during preparation are crucial for successful reconstitution, the conditions reported are rather different with respect to protease inhibitors, salt and lipid requirements. This might lead to the conclusion that the AcChR is easy to handle without special precautions. However, the data in the literature cited and their interpretations do not yet yield a consistent picture.

The data on AcChR single channel reconstitution published hitherto are aimed at showing similarities between reconstituted and native AcChRs. The reference for "native channel activity" is the electrocyte synapse. Up to now there are, for technical reasons, no data on single channel events of $T$. cal. electrocyte membranes. T. cal. AcChR expressed in oocyte membranes exhibits channel activity comparable to that of native muscle AcChR. In both cases it is not yet established what species - monomer or dimer or higher oligomers - is responsible for the measured single channel event. In addition, it is not known whether the oocyte modifies the $T$. cal. receptor protein and its function. Furthermore, the subsynaptic densities of Torpedo electrocytes hint at an interaction between the AcChR and cytoplasmic compounds such as the $\nu$-proteins and actin filament.

Here we present results demonstrating that there are distinct differences between oocyte-expressed T. cal. receptors (and native muscle AcChRs) and the isolated AcChR species in the reconstituted planar lipid membrane. Experimental strategies are proposed to improve the reconstitution procedure and to investigate regulatory processes such as receptor or lipid phosphorylation $[15,16]$. 
METHODS AND MATERIALS

\section{Preparation of $A c C h R s$}

Frozen tissue (200 g) from $T$. cal. electric organ (Pacific Biomarine, Venice, CA) was broken into small pieces and homogenized in $400 \mathrm{ml}$ of a buffer containing 10 $\mathrm{m} M$ piperazine- $N, N^{\prime}$-bis(2-ethanesulfonic acid) (PIPES), $\mathrm{pH} 7.1,5 \mathrm{~m} M$ ethylenediaminetetraacetic acid (EDTA), $10 \mathrm{~m} M \mathrm{NaN}_{3}, 5 \mathrm{~m} M$ iodoacetamide and $3 \mathrm{~m} M$ benzamidine). All chemicals were obtained from Sigma unless indicated otherwise. The protein was purified as described previously [11]. The receptor proteins was eluted from the affinity column with $10 \mathrm{~m} M$ PIPES containing $60 \mathrm{~m} M \mathrm{NaCl}, 0.5 \%$ (3-[(3-cholamidopropyl)-dimethylammonio]-1-propanesulfonate) (CHAPS), $5 \mathrm{mg} /$ $\mathrm{ml}$ soybean phospholipid (Avanti), and $50 \mathrm{~m} M$ Flaxedil or carbamoylcholine. The specific activity in the presence of detergent was $8 \mathrm{nmol} \alpha-\mathrm{Btx} / \mathrm{mg}$ protein, corresponding to 2 moles $\alpha$-Btx bound to one mole of receptor protein $\left(M_{\mathrm{r}} \approx\right.$ 290,000).

\section{Formation of vesicles}

\section{Lipid vesicles}

Soybean phosphatide extract containing 20\% lecithin (Avanti) was mixed with 10 wt\% cholesterol in hexane and dried under a stream of nitrogen in a round-bottom flask. The lipid film was resuspended in buffer, $100 \mathrm{~m} M$ Na-PIPES at a concentration of $25 \mathrm{mg} / \mathrm{ml}$ (bath sonication $1 \mathrm{~min}$ ) and subjected to 4-5 freeze-thaw cycles before storing the vesicles at $-80^{\circ} \mathrm{C}$. Before bilayer formation the $100 \mu 1$ samples were diluted about ten times and supplemented with the salts indicated in the figures. The described method of vesicle formation yields highly reproducible and stable bilayers, in contrast to lipid suspensions peeled from a thin lipid film with glass beads [11].

\section{Reconstituted AcChR vesicles}

Protein lipid vesicles were formed by conventional detergent dialysis; $20-32$ $\mathrm{mg} / \mathrm{ml}$ lipid in 2\% CHAPS were supplemented with $0.1 \mathrm{mg} / \mathrm{ml} \mathrm{AcChR}$-protein and dialysed against 500 sample volumes of buffer $\left(10 \mathrm{~m} M \mathrm{Na}^{+}\right.$-PIPES, pH 7.1, 150 $\mathrm{m} M \mathrm{NaCl}, 0.02 \% \mathrm{NaN}_{3}$ ) with four changes in three days. Alternatively the fast dilution technique [11] was tried.

\section{Formation of bilayers}

Bilayers were formed according to ref. 11. Before application of the protein-lipid vesicles to the cis side (where the agonist is applied) of the bilayer chamber, the sample was diluted 10 to 1000 times with lipid vesicles. The area of the bilayer was varied from $2 \times 10^{-4}$ to $3 \times 10^{-5} \mathrm{~cm}^{2}$. 
RESULTS

Patch clamp measurements at native muscle membranes suggest that there exists - beside relatively rare sublevels - one discrete open state with a defined conductance [3-6].

We have found that under conditions used mainly for reconstitution of AcChRs into planar bilayers (formation of bilayers via two vesicle-spread monolayers), discrete single conductance events are recorded with considerable differences in amplitude. Figure 1A shows a selection of such single-channel events (20-120 pS). In addition, these single channels can change into phases of rapidly fluctuating openings in the same measurement. In between these fluctuations, bursts of short single openings can be detected (Fig. 1B). The observed "fuzzy" conductance changes may be caused by receptor proteins which are modified by the reconstitution process or lack the adequate cellular environment. Although single channel events (Fig. 1) occur regularly in the reconstituted system, we frequently see larger conductance steps of, e.g., about $300 \mathrm{pS}$, probably caused by AcChR clusters opening cooperatively [11]. The assumption of cooperative multi-channels is supported by the fact that intermediate levels of conductance can also be seen in between. Therefore the data suggest a certain degree of variability in cluster cooperativity. As shown in Fig. 2, the channel activity can change from a burst with intermediate openings to a long opening with short closing gaps and then can

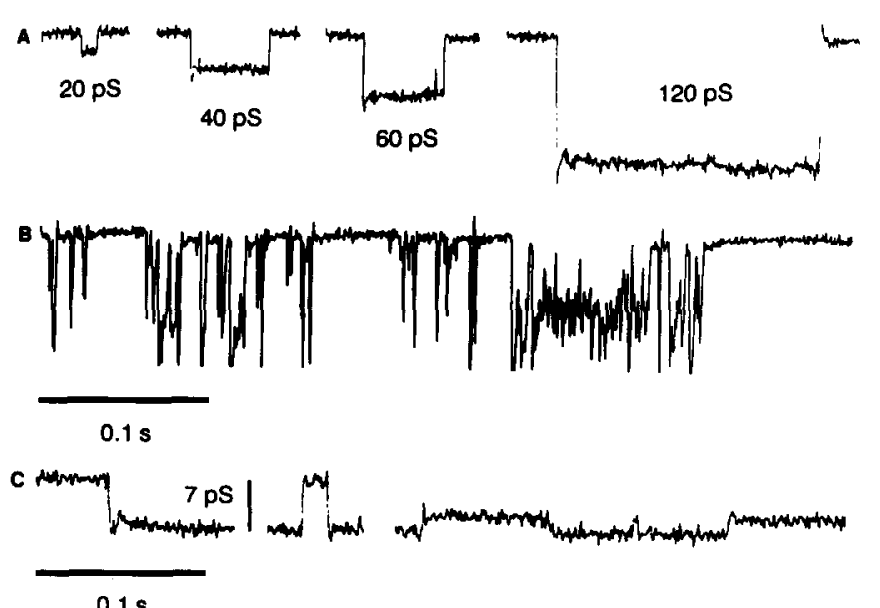

Fig. 1. Different channel amplitudes of reconstituted $T$. cal. AcChRs. Line A shows a selection of single channels from the same record. Between rectangular channels with long open times, short single channels and fuzzy bursts, line B, with rapidly changing amplitudes are seen. Membranes were formed in $450 \mathrm{~m} M$ $\mathrm{NaCl}, 1 \mathrm{~m} M$ EGTA, $10 \mathrm{~m} M$ PIPES (buffer A). The agonist was carbamoylcholine $(10 \mu M)$ and $E=150$ $\mathrm{mV}$. (C) At high resolution $(E=-250 \mathrm{mV}$ ) even very small channels are detected with about 7 and 2.5 pS. The channels have open times of one to several hundred ms. Buffer: same as in A and B. Agonist is suberoyldicholine $(2.5 \mu M)$. Channel opening is downwards. 


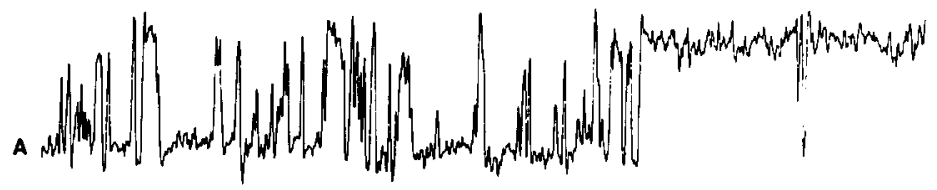

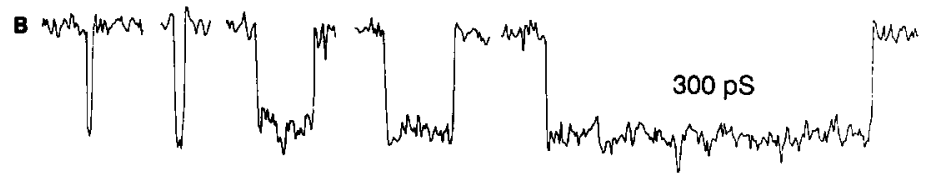

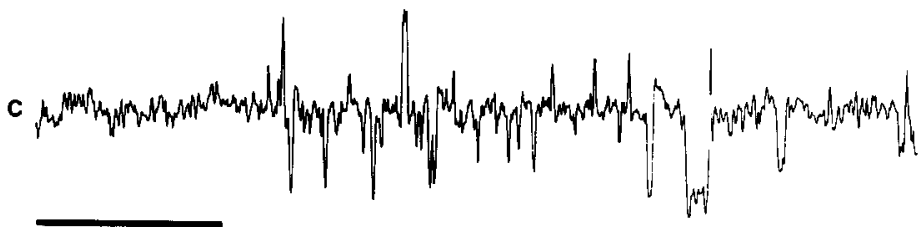

$0.05 \mathrm{~s}$

Fig. 2. Channel cluster. A, B and C show different sections of a continuous record. The channel events show the simultaneous opening and closing of a channel aggregate; the occurrence of "subniveaus" indicates that the coupling is not too strong. In line A a long burst is followed by a lasting opening with short closing gaps. Line B is an ensemble of channel closings (gaps) showing a great variety of closed times. (C) Two channel aggregates are active at the same time. One aggregate is closed most of the time whereas the other, open channel only shows short closing gaps. The mean conductance level corresponds to about $550 \mathrm{pS}$ from the baseline. Membranes were formed in buffer A. See legend to Fig. 1. The agonist was carbamoylcholine $(10 \mu M)$ and $E=+50 \mathrm{mV}$. Opening of the channels is upwards.
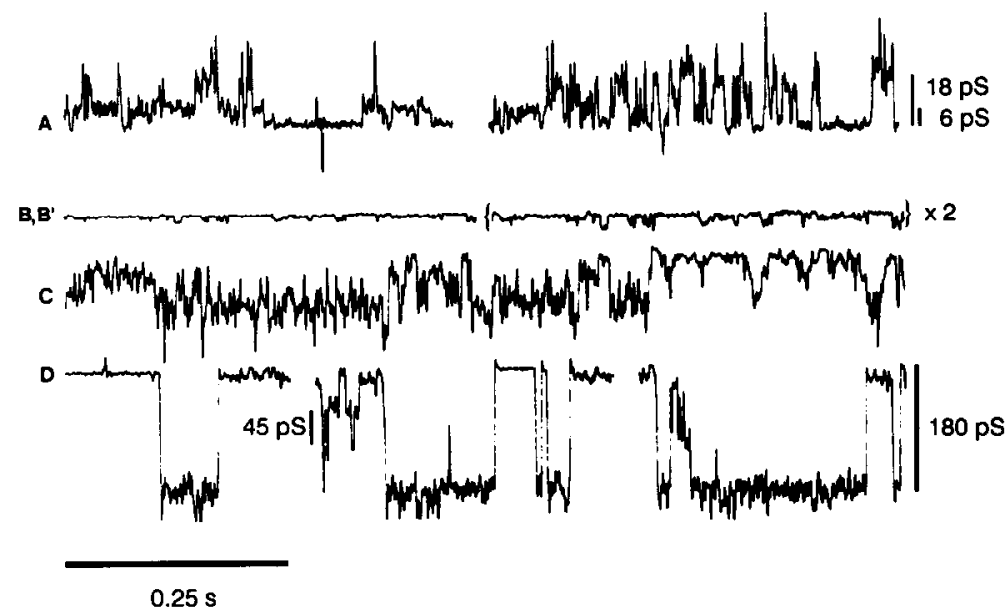

Fig. 3. Channel record in the presence of calcium. Buffer $\mathrm{A}$ was supplemented with $3.5 \mathrm{~m} M \mathrm{CaCl}_{2}$ after membrane formation. Channel fluctuations vanished after the addition of calcium. Channels could be reactivated by application of a high voltage $(>150 \mathrm{mV})$. Channels range from very small amplitudes (about $6 \mathrm{pS} ; \mathrm{A}, \mathrm{B}$ ) to several hundred $\mathrm{pS}$ (not shown). Line D shows a channel cluster of $180 \mathrm{nS}$ with substates of about $45 \mathrm{pS}$. The agonist is suberoyldicholine $(0.25 \mu M)$. Channels open upwards (A) and downwards (B-D). 


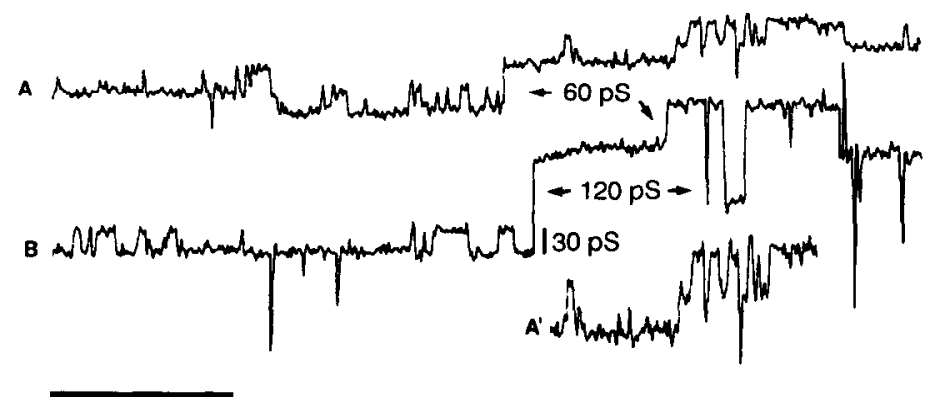

$0.1 \mathrm{~s}$

Fig. 4. Multiple channels. Sometimes it is possible to refer multiple openings of cooperative channels to more frequent "single" channels. The single channel conductance in this case is $30 \mathrm{pS}$. Buffer A was used at $2 \mu M$ carbamoylcholine. $\mathrm{A}^{\prime}$ is a two-fold magnification of the corresponding segment in $\mathrm{A}$. Channels open in the upward direction.

change to longer closed states, too. For instance, in line C of Fig. 2 two "aggregates" are active at the same time, one of them being closed most of the time, while the second is open and closes in short gaps in between.

Though the multiplicity of step sizes normally does not allow the determination of a "unit channel", sometimes it is possible to refer larger conductance steps to small "single" channels, as represented in Fig. 3. Here $30 \mathrm{pS}$ channels dominating the conductance changes alternate with steps of 60 and $120 \mathrm{pS}$. The record is, however, not sufficient to determine structure-function relationships. The data nevertheless demonstrate cooperative gating behavior.

After the addition of $\mathrm{Ca}^{2+}$ (to yield a concentration of 2-5 $\mathrm{mM}$ ) to the cis side (where the protein was added), the channel activity decreased to zero at moderate voltages. But after application of higher voltages $(\geqslant 150 \mathrm{mV})$ the channels were reactivated (Fig. 4). Line $A$ in Fig. 4 shows a fuzzy burst of an 18 pS channel. As in the measurements without $\mathrm{Ca}^{2+}$ small channels (about $6 \mathrm{pS}$ ) can be detected either as sublevels of a burst (A) or as single channels (B). In addition, fluctuating conductance changes (line $\mathrm{C}$ ) as well as aggregates (D) occur also in the presence of $\mathrm{Ca}^{2+}$. In contrast to $\mathrm{Ca}^{2+}$-free medium, the channels at higher $\mathrm{Ca}^{2+}$ concentrations often close directly to the baseline, indicating increased cooperative behavior of the conductance changes.

\section{DISCUSSION}

\section{Activation of AcChRs}

In the synaptic gap of cholinergic nerve muscle contacts $(\approx 20-50 \mathrm{~nm})$, nerve excitation transiently rises the $\mathrm{AcCh}$ concentration from $<10^{-7}$ to about $10^{-4}$ mol $/ 1$ in a fraction of a millisecond (see e.g. ref. 17). This rapid kinetics can hardly be simulated at a receptor channel reconstituted in a planar bilayer. At the planar bilayer an unstirred layer of a thickness of 0.1 to $0.5 \mathrm{~mm}$ exists, depending on the 


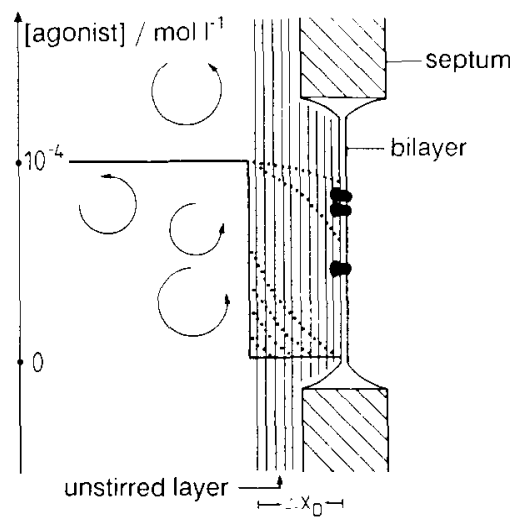

Fig. 5. Geometry of agonist application to the planar bilayer system. The unstirred layer causes an increase of agonist concentration near the reconstituted receptor by diffusion only. As the desensitized state has a higher binding constant for $\mathrm{AcCh}\left(10^{-6} \mathrm{M}\right)$ compared to the active conformation $\left(10^{-4} \mathrm{M}\right)$ the receptor equilibrium will be shifted to the desensitized state, before an activating concentration of agonist is reached at the membrane surface. The diffusion time of one-dimensional diffusion is given by $t_{\mathrm{D}}=\left\langle x_{\mathrm{D}}^{2}\right\rangle / 2 D=5-500 \mathrm{~s}\left(D \approx 10^{-5} \mathrm{~cm}^{2} / \mathrm{s}, x_{\mathrm{D}}=0.01-0.1 \mathrm{~cm}\right)$.

velocity of stirring (Fig. 5). The diffusion time for applied AcCh to reach the planar bilayer is estimated to be a few seconds to one minute. Stopped flow measurements show that the time constant of receptor desensitization is in the range of $100 \mathrm{~ms}$ to 1 $s$ [17]. Therefore, in the best case the very end of an activation phase of the receptor channel after AcCh application can be expected in a planar bilayer experiment. Up to now we, as well as all other investigators, have not seen a clear transient activation of AcChRs in the planar bilayer. Instead, we have concentrated on recording channel events probably arising from the desensitized state. It is likely that all single channel data of AcChRs in planar bilayers published so far refer to largely desensitized receptors. Normally, the agonist $(0.1 \mu M)$ was added to the protein lipid vesicles before bilayer formation. According to Schindler et al. [1] the agonist may retain the viability of the receptor protein during bilayer formation. When membrane fragments or vesicle-reconstituted receptors are fused with a preformed lipid bilayer, agonist is added from the beginning, too $[9,10]$. No AcChR activity was detected after fusion of AcChR vesicles to planar lipid bilayers containing decane [10]. Boheim et al. [9] report the successful fusion of vesicles containing purified AcChRs into planar steroyl-myristoyl-phosphatidylcholine (SMPC) bilayers; here, too, the observed channel openings probably arose from the desensitized receptor state. Another group found that AcChR channels vanish shortly after the formation of a vesicle patch; this observation was also referred to receptor desensitization [14]. In contrast, when the vesicle/planar bilayer fusion method $[9,10]$ is used, the first channel events are detected from several minutes up to half an hour after formation. Furthermore, at patched vesicles we observe channel events during a time interval of several minutes, after vesicle patch 
formation. An actual channel activation immediately after addition of agonist to reconstituted AcChRs has been reported [7,8,12]. However, Nelson et al. [8] regret that they could activate AcChRs only when they had seen a basic conductivity before the addition of agonist. In comparison to the references mentioned, it is therefore not surprising that the channel events we have found normally open "spontaneously", without direct correlation to the addition of agonist .

In agreement with the patch clamp measurements reported by Jackson [18], we occasionally find rare channel events of the reconstituted AcChRs even without agonist added. The rate of appearance of reconstituted AcChR channels induced with agonist is already rather low (probably due to receptor desensitisation). The variability of the recorded channels is rather high. At present, it is therefore not possible to derive a reaction model from the reconstitution data to describe the activation and desensitization kinetics of the reconstituted $T$. cal. AcChR. Up to now, there are still no single channel data available from the electric organ synapse, which could serve as a reference for the experiments with reconstituted AcChRs in planar membranes. Patch clamp measurements at muscle membranes are only partially suitable as reference, because there the desensitization is not so pronounced and receptor organization is different, especially in cultured cells.

\section{Channel conductivity}

A basic aim of protein/bilayer reconstitution is the establishment of a defined structure-function relationship. The patch clamp measurements of cellular membranes show apparently only one distinct open state. Therefore the data of the reconstituted AcChRs were at first analysed with a simple two-state model, too. A single channel event is usually thought to reflect the smallest channel unit which can be detected from a dilute sample.

However, at least two problems have to be considered which hamper the interpretation of our present channel data: sublevels and protein aggregation. Sublevels of AcChR have already been described in patch clamp analyses [5], and F. Sachs asks: "Is the acetylcholine receptor a unit-conductance channel?" [19]. There is no doubt that the reconstitution procedures can lead to the formation of additional conformational states (not allowed in the native environment), some of them being active in ion conduction. Actually this is the impression when analysing our channel data in terms of defined molecular species, monomer, dimers or higher oligomers of reconstituted AcChRs. Our data do not contradict the patch clamp results, but a consistent interpretation in terms of substates and defined channel species is at present very difficult. As long as the $\Lambda c C h R$ channel activity cannot be titrated quantitatively as a function of protein and agonist concentration, the determination of open and closed times remains unresolved.

Ten years ago, Anholt et al. [20] found that there is a limit to receptor dilution. They could not produce vesicles with a protein/lipid ratio below $1: 16(\mathrm{wt} / \mathrm{wt}$ ). This result indicates that during dialysis for detergent removal there is a partition of constituents; the receptor proteins are already aggregated in the vesicles. Recently, we have found that at lipid/detergent ratios near the vesicle/micelle transition a 


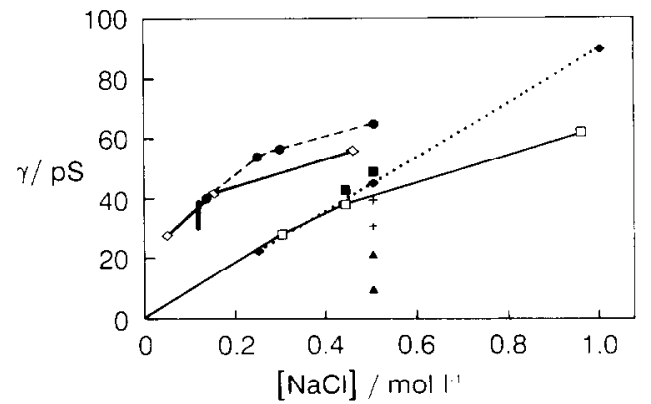

Fig. 6. Single channel conductances. The different single channel conductances, which have been published by different authors, are plotted as a function of $\mathrm{NaCl}$ concentration. The references are given in brackets. (๑) Reconstituted vesicle patch, $1 \mathrm{~m} M \mathrm{Ca}^{2+}, 1 \mathrm{~m} M \mathrm{Mg}^{2+}$ [14]; [O, ] patch clamp, no $\mathrm{M}^{2+}$ [5,6]; (๑) reconstituted synaptosomes (microsacs), $1 \mathrm{~m} M \mathrm{Ca}^{2+}, 1 \mathrm{mM} \mathrm{\textrm {Mg } ^ { 2 + }}$ [7]; (+) (dimers),

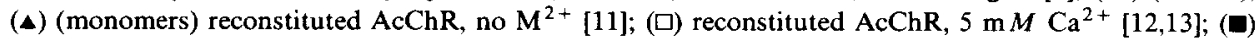
as before, but different experiment. For easier comparison of the data we have taken the concentration values instead of the activity.

phase separation takes place in an aqueous suspension of lecithin- $\beta$-octylglucoside (unpublished data).

When AcChR-lipid-detergent solutions are centrifuged on a sucrose gradient (containing no detergent), AcChRs can be separated into monomers $\left(M_{\mathrm{r}} \approx 290,000\right)$, dimers and oligomers (data not shown). 'This implies that the proteins are not incorporated into the lipid vesicles when the detergent is removed too fast. Schindler et al. [11] have used a specific procedure (named fast dilution) as a means to get a homogeneous vesicle population with not more than one protein per vesicle. According to our results, in the sucrose gradient this favorable result is not seen. In addition, the products of the fast dilution technique have never been analysed with respect to their structural composition.

Experiments on the reconstitution of erythrocyte band 3 protein revealed that there too, protein function and aggregation depend on the lipid-detergent composition and on the way of vesicle bilayer formation [21].

Anyhow, some publications give the impression that one type of channel event can be related to one reconstituted AcChR species. However, the heterogeneity of the data from different groups is more similar to the variability of channels we have seen. In Fig. 6, single channel conductances from several AcChR publications are plotted as a function of $\mathrm{NaCl}$ concentration.

It can be seen that the patch clamp data already vary between 31 and $39 \mathrm{pS}(125$ $\mathrm{m} M \mathrm{NaCl}$ ), indicating that a certain variance in conductance appears as a natural property of the channel. Since no single channel data are available for the AcChR in the intact electrocytes, it cannot be excluded that the AcChR from $T$. cal., which is generally used for reconstitution experiments, shows a high variance of conductance states in its natural environment, too. 
According to patch clamp measurements, the conductance of a single channel is in the range of $75 \mathrm{pS}$ at $500 \mathrm{mM} \mathrm{NaCl}$ and is reduced to about $50 \%$ in the presence of divalent cation at concentrations of $1-5 \mathrm{mM}$. Most of the data from reconstituted $\mathrm{nAcChRs}$ do not fit into this physiological data frame: the patch data from reconstituted vesicles have too high a conductance for the presence of $2 \mathrm{mM}$ divalent cations [14]. The channel data of Schindler and Quast [7] show a linear dependence on $\mathrm{NaCl}$ concentration. This is expected for a large water-filled pore. More recent models assume cation binding sites at the channel mouth and, accordingly, the data are analysed in terms of the Michaelis-Menten formalism [22]. The single channel conductance given by Schindler et al. [11] for reconstituted receptors appears to be too low with respect to the absence of divalent cations. Furthermore, the $190 \mathrm{pS}$ channels in $1 \mathrm{M} \mathrm{K}^{+}$, the conductance of which is reduced to $80 \mathrm{pS}$ in the presence of $6 \mathrm{~m} M$ divalent ions, are rather peculiar; no such effect was seen with $\mathrm{Na}^{+}(90 \mathrm{pS})$ instead of $\mathrm{K}^{+}[9]$.

Different channel levels were also observed [11]. Beside the $40 \mathrm{pS}$ level there was a $30 \mathrm{pS}$ channel resulting from reconstituted receptor dimers and 20 and $9 \mathrm{pS}$ channels after disulfide-bond reduction, which yields the receptor monomer. The probability of the different channel levels was dependent on the $\mathrm{K}^{+} / \mathrm{Na}^{+}$ratio. In summary, comparison of the different single channel conductance values suggests that a thorough and critical reworking of $\mathrm{AcChR}$ reconstitution is needed.

Schindler et al. [11] also report on large channel events. We observe similar results. These channels were suggested to result from the cooperative opening and closing of AcChR aggregates. The larger single channels indicate that in lipid bilayers receptor aggregation is a spontaneous process. In the native membrane some additional factors, probably the $\nu$-proteins, may stabilize a particular oligomer organization in the postsynaptic membranes. Cooperative opening of multiple channels has also been reported for $\mathrm{Cl}^{-}$-channels of epithelia [23,24].

At variance with the data of Schindler et al. [11], the larger channels arising from AcChR aggregates also appear in the absence of calcium. It seems that the presence of $\mathrm{Ca}^{2+}$ stabilizes receptor conformations such that the activation of the channel clusters now is voltage dependent.

\section{CONCLUSION}

Although the $T$. cal. nAcChR channels sometimes appear as multiples of a basic element, a clear assignment of the channel amplitudes to defined receptor species (monomer, dimer, etc.) cannot be made yet. The variability of conductance states does not permit a "normal channel" to be distinguished from a substate or from an odd conformation. The main reason for the unusual channel appearances of reconstituted AcChR proteins is most likely the enormous tendency of the AcChR to aggregate in a lipid environment, as was also found in biochemical studies [25].

In order to get only a few, and therefore unaggregated, receptor proteins in the planar bilayer, the vesicles must contain preferably only one protein per vesicle. Therefore, a reconstitution procedure has been developed which avoids the miscibil- 
ity gap (and thus phase separation) in a particular detergent/lipid system (unpublished results). The lipid bilayer vesicles resulting from the detergent/lipid phase separation may be characterized by electron microscopy. In particular, the number of protein molecules in the vesicle membrane may be determined. These vesicles should be useful both for planar bilayer measurements and for the direct patch clamp. It should then be possible to investigate how the $\mathrm{nAcChR}$ is activated by $\mathrm{AcCh}$ and whether the channel function can be also controlled by second messengers, e.g. cAMP-dependent kinase [15].

\section{ACKNOWLEDGFMFNT}

We thank Professor H.W. Chang for her work on AcChR-protein isolation, Professor H. Mautner for instructive discussions and B. Büddemann and A. Tiemann for excellent technical assistance. We gratefully acknowledge financial support by the Deutsche Forschungsgemeinschaft (SFB 223/D3 to E.N.).

\section{REFERENCES}

1 M. Noda, H. Takahashi, T. Tanabe, M. Toyosato, Y. Furutani, T. Hirose, M. Asai, S. Inayama, T. Miyata and S. Numa, Nature (London), 299 (1982) 793.

2 M. Noda, H. Takahashi, T. Tanabe, M. Toyosato, S. Kikyotani, Y. Furutani, T. Hirose, H. Takashima, S. Inayama, T. Miyata and S. Numa, Nature (London), 302 (1983) 528.

3 E. Neher and B. Sakmann, Nature (London), 260 (1976) 799.

4 P. Adams, T.M. Dwyer and B. Hille, J. Gen. Physiol., 75 (1980) 493.

5 D. Colquhoun and B. Sakmann, J. Physiol., 369 (1985) 501.

6 A.D. Dani and G. Eisenman, J. Gen. Physiol., 89 (1987) 959.

7 H. Schindler and U. Quast, Proc. Natl. Acad. Sci. USA, 77 (1980) 3052.

8 N. Nclson, R. Anholt, J. Lindstrom and M. Montal, Proc. Natl. Acad. Sci. USA, 77 (1980) 3057.

9 G. Boheim, W. Hanke, F.J. Barrantes, H. Eibl, B. Sakmann, G. Fels and A. Maelicke, Proc. Natl. Acad. Sci. USA, 78 (1981) 3586.

10 W. Hanke, Bioelectrochem. Bioenerg., 12 (1984) 341.

11 H. Schindler, F. Spilleke and E. Neumann, Proc. Natl. Acad. Sci. USA, 81 (1984) 6222.

12 P. Labarca, J. Lindstrom and M. Montal, J. Gen. Physiol., 83 (1984) 473.

13 M. Montal, P. Labarca, D.R. Fredkin, B.A. Suarez-Isla and J. Lindstrom, Biophys. J., 45 (1984) 165.

14 D.W. Tank, R.L. Huganir, P. Greengard and W.W. Webb, Proc. Natl. Acad. Sci. USA, 80 (1983) 5129.

15 R.L. Huganir, A.H. Delcour, P. Greengard and G.P. Hess, Nature (London), 321 (1986) 774.

16 R. Kiehl, M. Varsanyi and E. Neumann, Biochem. Biophys. Res. Commun., 147 (1987) 1251.

17 J.P. Changeux, A. Devillers-Thiéry and P. Chemouilli, Science, 225 (1984) 1335.

18 M.B. Jackson, Proc. Natl. Acad. Sci. USA, 81 (1984) 3901.1

19 F. Sachs in B. Sakmann and E. Neher (Eds.), Single-Cliannel Recording, Plenum Press, New York, 1983 , p. 365.

20 R. Anholt, J. Lindstrom and M. Montal, J. Biol. Chem., 256 (1981) 4377.

21 U. Scheuring, K. Kollewe, W. Haase and D. Schubert, J. Membr. Biol., 90 (1986) 123.

22 J.A. Dani and G. Eisenman, Biophys. J., 45 (1984) 10.

23 V.I. Geletyuk and V.N. Kazachenko, J. Membr. Biol., 86 (1985) 9.

24 M.E. Krouse, G.T. Schneider and P.W. Gage, Nature (London), 319 (1986) 58.

25 V. Volz, Diploma Thesis, University of Bielefeld, 1987. 